\title{
Prediction outcome probabilities as determinants of choice reaction time*
}

\author{
CHARLES P. WHITMAN and E. SCOTT GELLER $\dagger$ \\ Virginia Polytechnic Institute and State University, Blacksburg, Virginia 24061
}

\begin{abstract}
In a two-stimulus two-response choice reaction time (RT) task in which Ss made stimulus predictions, the probability of a correct prediction was manipulated between Ss. The magnitude of the difference in RT to correctly and incorrectly predicted stimuli (i.e., the prediction outcome effect) was an increasing function of the probability of a correct prediction. This finding was primarily due to a reliable decrease in RT to correctly predicted stimuli as the probability of a correct prediction increased, since RT to incorrectly predicted stimuli was not affected by prediction outcome probability. These results were interpreted as partially supporting a continuous expectancy notion which involves facilitory and inhibitory mechanisms which are differentially influenced by the probability of a correct prediction.
\end{abstract}

Choice reaction time (RT) experiments in which Ss predict which stimulus will occur prior to a stimulus presentation have demonstrated reliably shorter latencies to correctly anticipated stimuli and to the more probable of two stimuli (e.g., Geller, Whitman, \& Farris, 1972; Hinrichs \& Craft, 1971; Whitman \& Geller, 1972). A model which has been used to explain the independent effects of stimulus probability and prediction outcome on choice RT was derived from the continuous expectancy notions of Geller and Pitz (1970). One of their basic assumptions was that Ss' expectancy for a correctly predicted stimulus was a direct function of $\mathrm{Ss}^{\prime}$ confidence that their stimulus prediction would be correct. Thus, the results of recent choice RT experiments were explained by assuming Ss' confidence in a prediction to be greater when the more probable stimulus was predicted (Geller, Whitman, Wrenn, \& Shipley, 1971; Geller et al, 1972) and when the immediately preceding prediction had been correct (Whitman \& Geller, 1971, 1972).

The present study was designed to determine the influence of the relative frequency of correct and incorrect predictions on choice RT. As the probability of a correct prediction increases, so should Ss' confidence in the correctness of their stimulus predictions, and consequently their expectancy for the predicted stimulus should be augmented. A greater expectancy in this case should effect increased facilitation of reactions to correctly predicted stimuli and greater inhibition of reactions to incorrectly predicted stimuli. Thus, the magnitude of the prediction outcome effect is hypothesized to be an increasing function of the probability of a correct prediction. That is, choice RT facilitated by a correct stimulus prediction

*This research was partially supported by the Small Grants Program of the College of Arts and Sciences, Virginia Polytechnic Institute and State University.

†Reprint requests should be sent to E. Scott Geller, Department of Psychology, Virginia Polytechnic Institute and State University, Blacksburg, Virginia 24061. should be a decreasing function of the probability of a correct prediction, while choice RT inhibited by an incorrect prediction should be an increasing function of the probability of a correct prediction.

The basic methodology of the present study has been used previously in a probability learning paradigm to evaluate the effects of correct predictions on Ss' prediction strategies. Specifically, the present research was a between-Ss application of the noncontingent success design used by Yellott (1969). With this method, the probability that a given prediction would be correct was independent of which stimulus Ss predicted. That is, letting $E_{1}$ and $E_{2}$ represent the two stimulus events and $A_{1}$ and $A_{2}$ represent $S s^{\prime}$ predictions of $E_{1}$ and $E_{2}$, respectively, $P\left(E_{1} \mid A_{1}\right)$ was equal to $P\left(E_{2} \mid A_{2}\right)$ and was varied between Ss.

\section{METHOD}

The $50 \mathrm{Ss}$ were volunteers from introductory psychology classes at Virginia Polytechnic Institute and State University. The Ss were randomly assigned to one of five groups in which the probability of a correct prediction was $.10, .30, .50, .70$, or .90. Three $\mathrm{Ss}$ who made more than 15 identification errors (i.e.. $5 \%$ ) were dropped from the analysis and replaced. No more than six Ss in any one group were of the same sex.

The two-stimulus two-response choice reaction task required Ss to make stimulus predictions prior to each of the 300 stimulus presentations. The stimuli were the symbols $\sqcup$ and $\Pi$ (verbally labeled "up" and "down," respectively), which were presented by a $1.5 \times 2.5 \mathrm{~cm}$ digital readout. Five $S s$ in each group pulled a microswitch trigger in a right handle to identify an "up" and a left-handle trigger to identify a "down," while the remaining five $S s$ used the reverse $S-R$ relationship (i.e.. a left response to an "up" and a right response to a "down"). After making a verbal stimulus prediction, Ss pressed a lever switch corresponding to the prediction. Logic equipment combined the result of this response with information from a paper tape to determine whether the predicted or nonpredicted stimulus would occur. In the event that S's verbalized prediction and switch press did not correspond, $E$ informed $S$ of the error and instructed him to remake a verbal prediction and switch-press combination. The spring-lever switch for an "up" prediction was mounted on an inclined panel $8 \mathrm{~cm}$ above the switch for a "down" prediction. Following S's stimulus prediction, E sounded a warning buzzer which preceded the stimulus 
Table 1

Error Rate as a Function of the Probability of a Correct Prediction and the Prediction. Outcome

\begin{tabular}{cccc}
\hline $\begin{array}{c}\text { Correct } \\
\text { Prediction } \\
\text { Probability }\end{array}$ & $\begin{array}{c}\text { Error } \\
\text { Rate }\end{array}$ & \multicolumn{2}{c}{$\begin{array}{c}\text { Prediction Outcome } \\
\text { and Error Rate }\end{array}$} \\
\cline { 2 - 4 } & Correct & Incorrect \\
\hline .10 & 2.17 & 3.67 & 2.00 \\
.30 & 1.23 & 1.11 & 1.33 \\
.50 & 1.40 & 1.33 & 1.47 \\
.70 & 1.80 & 0.95 & 3.78 \\
.90 & 1.57 & 0.85 & 8.00 \\
\hline
\end{tabular}

presentation by a variable time interval of between 1 and 2 sec. The interstimulus interval was approximately $8 \mathrm{sec}$.

Three binary sequences of 300 points each, a 90-10, a 70-30. and a 50-50. were determined by filtering a uniform random-number generator on an IBM computer. The Ss were informed that the stimulus sequence was predetermined on a punched paper tape (the tape actually controlled prediction outcome). The task instructions emphasized both speed and accuracy in identifying the stimuli.

\section{RESULTS AND DISCUSSION}

\section{Choice Reaction Errors}

Trials on which Ss' choice response either anticipated the stimulus presentation or was incorrect were categorized as errors. The mean error rate is reported in Table 1 as a function of correct prediction probability and prediction outcome. As evident in Table 1, the overall error rate was not greatly influenced by the probability of a correct prediction. A Kruskal-Wallis test for the five independent samples indicated no reliable differences. $p>.20$.

Columns 3 and 4 of Table 1 present the error rate on trials with correct and incorrect prediction outcomes, respectively. For correct prediction probabilities of 10 , .30. and .50, the Wilcoxon test for two correlated samples indicated no effect of prediction outcome on error rate, all ps $>.10$. However, for the correct prediction probabilities .70 and .90 , the Wilcoxon test indicated significantly higher error rates in choice reactions to incorrectly predicted stimuli, $\mathrm{p}<.01$. Thus, when the probability of a correct prediction outcome was .10 . .30. or .50 . the tendency to make errors was not influenced by prediction outcome. As the probability of a correct prediction outcome increased from .50 to .90 . the error rate for choice reactions to incorrectly predicted stimuli reliably increased.

Considering that error rate of choice reactions to incorrectly predicted stimuli indicates a tendency to respond as though the expected stimulus occurred when in fact it did not. the increase in error rate to incorrectly predicted stimuli as a function of the probability of a correct prediction reflected Ss' increased expectancy for the predicted stimulus. Moreover, the result that error rate of choice reactions to correctly predicted stimuli was essentially constant suggested that Ss anticipate the predicted stimulus even when the correct prediction probability was .10 or .30 .

\section{Choice Reaction Time}

Each RT was categorized according to a correct or incorrect prediction outcome and then the group means for each category were computed by averaging the category means of each group of Ss. Trials on which $\mathrm{S}$ made incorrect or anticipatory choice responses were excluded from the analysis and did not exceed $5 \%$ for any $S$.

As depicted in Fig. 1, choice RT to correctly predicted stimuli was a monotonic decreasing function of the probability of a correct prediction, but choice RT to incorrectly predicted stimuli was not consistently influenced by the probability of a correct prediction. An overall analysis of variance (ANOVA), a 5 (probabilities) by 2 (prediction outcomes), indicated a significant interaction $[F(4,45)=9.01 . p<.001]$. The ANOVA of the latencies to incorrectly predicted stimuli indicated no main effect of probability $(\mathrm{F}<1)$, while the ANOVA of latencies to correctly predicted stimuli indicated a reliable effect of probability $[F(1,45)=3.71, p<.025]$. Tukey's HSD test indicated that the "reversed" effect of

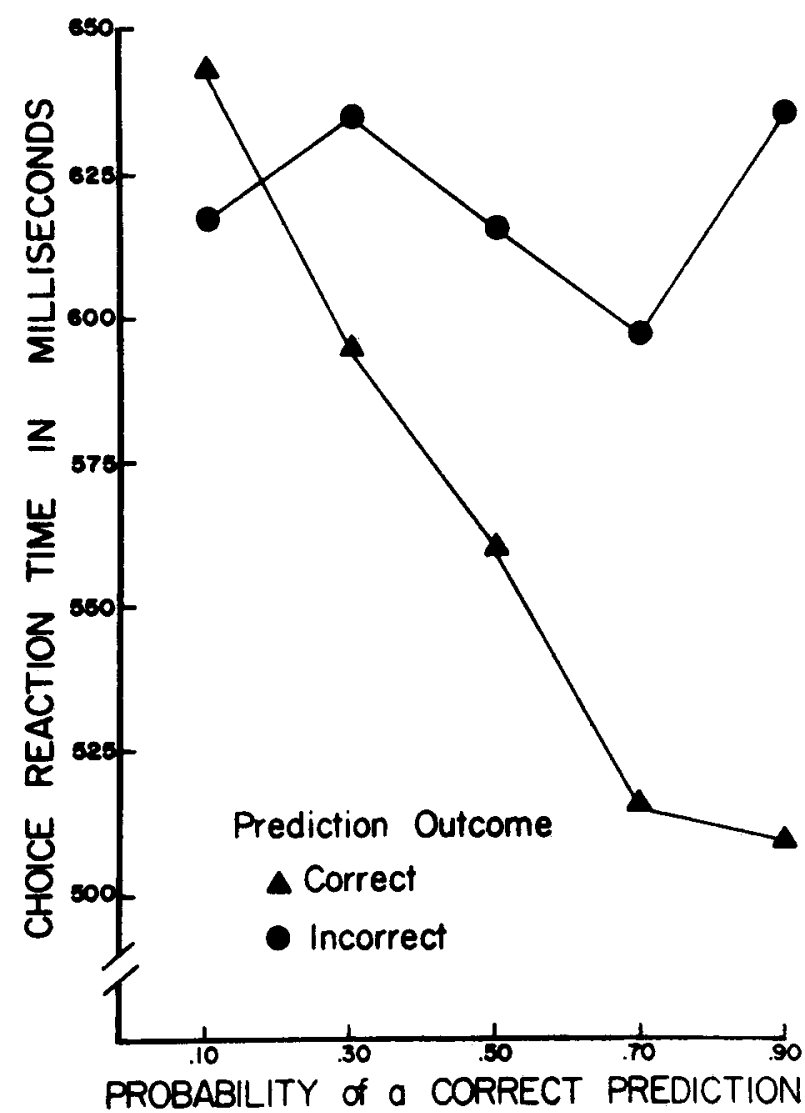

Fig. 1. Choice RT as a function of five levels of the between-Ss variable: the probability of a correct prediction. 
prediction outcome for correct prediction probability .10 was not significant $(.25>\mathrm{p}>.10)$.

To determine whether the influence of the probability on choice RT was significantly confounded by practice effects (i.e., trials) or by stimulus frequency effects (i.e., Ss' prediction sequences), two other analyses were completed. One analysis considered trial blocks of 50 trials as a variable. A 5 (probabilities) by 6 (trials blocks) by 2 (prediction outcomes) ANOVA indicated a main effect of trial blocks $[F(5,225)=12.37, p<.001]$, but trial blocks did not interact with either groups or prediction outcome (all Fs $<1.50$ ). An analysis of Groups .10 and .90 studied the possibility that Ss' prediction strategies resulted in stimulus frequency imbalances and thus biased the observed effects of prediction outcome. Since the direction of any resulting frequency imbalance was a function of S's particular prediction strategies, the more probable stimulus alternative was determined independently for each S. The mean frequency imbalance over the $20 \mathrm{Ss}$ of Probability Conditions .10 and .90 was only $7 \%$ (i.e., the mean probability for the more probable stimulus was 53.3). An ANOVA of 2 (probability groups: .10 or .90) by 2 (prediction outcomes: correct or incorrect) by 2 (stimuli: more or less frequent) showed no main effect of stimulus and no reliable interactions involving stimulus ( $\mathrm{ps}>.10$ ).

The continuous expectancy notions proposed in the present report assumed that stimulus expectancy increased directly with the proportion of correct predictions. Thus, the degree of RT facilitation due to correctly predicting a stimulus should be a direct function of correct prediction probability. Correspondingly, the degree of response inhibition following an incorrectly predicted stimulus was assumed to be a direct function of the expectancy for the predicted stimulus. In addition to accounting for the well-established result that RT to correctly predicted stimuli is shorter than RT to incorrectly predicted stimuli when the two prediction outcomes are approximately equiprobable, this two-process model accounts for the finding that choice RT to correctly predicted stimuli was a decreasing function of the probability of a correct prediction. However, the observation that RT to incorrectly predicted stimuli was not reliably influenced by the probability of a correct prediction implies that either the hypothesized inhibitory mechanism does not exist or that inhibition and facilitation are differentially influenced by expectancy changes due to the manipulation of the probability of a correct prediction. Thus, some additions or deletions in the continuous expectancy theory are needed to account for the present RT data.

\section{Superstitious Prediction Patterns}

Yellott (1969) reported that in a noncontingent success, probability learning design, 18 of 50 Ss exhibited superstitious solutions by predicting patterns of stimuli when the probability of a correct prediction was 1.0. However, it should be noted that Yellott also collected 200 trials of prediction data with the probability of a correct prediction being .80 and did not report any evidence of superstitious behavior. Furthermore, Yellott's concluding comments consider a model of prediction behavior which maintains that incorrect prediction outcomes disrupt Ss' memory of their position in a prediction pattern. In the present research, only 2 of $10 \mathrm{Ss}$ in Group .90 exhibited recognizable prediction patterns. One S's solution was to always predict the stimulus which occurred on the preceding trial. Letting $a_{1}$ and $a_{2}$ represent S's predictions of the two stimulus events $e_{1}$ and $e_{2}$, the prediction-stimulus pattern of this $S$ was $a_{1} e_{2}, a_{2} e_{2}$, $a_{2} e_{2}, a_{2} e_{1}, a_{1} e_{1}, \ldots$ The second $S$ always predicted that stimulus alternative which did not occur on the preceding trial (e.g., $a_{1} e_{1}, a_{2} e_{2}, a_{1} e_{1}, a_{2} e_{2}, a_{1} e_{2}, a_{1} e_{1}$, $a_{2} e_{2}, a_{1} e_{1}, \ldots$.). It may be important to note that both of these apparent patterns were dependent om the preceding stimulus rather than on the preceding prediction. In this respect, the prediction patterns observed with 90\% "partial reinforcement" in the present study were quite different from the patterns Yellott observed with "continuous reinforcement."

\section{REFERENCES}

Geller, E. S., \& Pitz, G. F. Effects of prediction, probability, and run length on choice reaction speed. Journal of Experimental Psychology, 1970, 84, 361-367.

Geller, E. S., Whitman, C. P., Wrenn, R. F., \& Shipley, W. G. Expectancy and discrete reaction time in a probability reversal design. Journal of Experimental Psychology, 1971, 90, 113-119.

Geller, E. S., Whitman, C. P., \& Farris, J. C. Probability discrimination indicated by stimulus predictions and reaction speed: Effects of S-R compatibility. Journal of Experimental Psychology, 1972, 93, 404-409.

Hinrichs, J. V., \& Craft, J. L. Verbal expectancy and probability in two-choice reaction time. Journal of Experimental Psychology, 1971, 88, 367-371.

Whitman, C. P., \& Geller, E. S. Prediction outcome, S-R compatibility and choice reaction time. Journal of Experimental Psychology, 1971, 91, 299-304.

Whitman, C. P., \& Geller, E. S. Sequential effects of stimulus probability and prediction outcome on choice reaction time. Journal of Experimental Psychology, 1972, 93, 373-378.

Yellott, J. I., Jr. Probability learning with noncontingent success. Journal of Mathematical Psychology, 1969, 6, 54 1-575.
(Received for publication August 14, 1972; revision received October 18,1972 .) 